\title{
Ovejas Pelibuey sincronizadas con progestágenos y apareadas con machos de razas Dorper y Katahdin bajo condiciones estabuladas: producción de la oveja y crecimiento de los corderos durante el período predestete
}

\author{
Pelibuey ewes synchronized with progestagens and mated with rams from Dorper and Katahdin \\ breeds under feedlot conditions: ewe production and lamb growth during the pre-weaning period \\ U Macías-Cruz ${ }^{a}$, FD Álvarez-Valenzuela ${ }^{a}$, HA Olguín-Arredondo ${ }^{b}$, \\ L Molina-Ramírez ${ }^{\mathrm{c}}$, L Avendaño-Reyes ${ }^{\mathrm{a}^{*}}$ \\ ${ }^{a}$ Instituto de Ciencias Agrícolas, Universidad Autónoma de Baja California, Baja California, México. \\ b Depto. de Ciencias Veterinarias, ICB, Universidad Autónoma de Ciudad Juárez, Chihuahua, México. \\ ${ }^{\mathrm{c}}$ Bachillerato Tecnológico Agropecuario No 41, Baja California, México.
}

\begin{abstract}
SUMMARY
The aim of this study was to evaluate the productivity of 253 Pelibuey $(\mathrm{Pb})$ ewes lambed over a period of four years (2005-2008), which were synchronized with progesterone and PMSG and mated to Pb, Dorper ( Dr), and Katahdin (Ka) rams under confinement. Additionally, birth and weaning weight, and pre-weaning daily weight gain (GDP) of 499 lambs born from these crosses were also evaluated. A linear model including the effects of year of lambing (block), sire breed, type of lambing, sex and their interactions, was used to analyze birth and weaning weight, and GDP of lambs. The same model, without the effect of sex was performed to analyze ewe productivity. Birth weight and mortality percentage at $30 \mathrm{~d}$ post-lambing were similar $(\mathrm{P}>0.05)$ among lamb genotypes, but weaning weight and GDP were higher $(\mathrm{P}<0.05)$ in lamb's crosses from $\mathrm{Dr}$ than in those pure $\mathrm{Pb}$ and crosses from Ka. The interaction type of lambing $\times$ sex affected $(\mathrm{P}<0.05)$ pre-weaning performance. At $30 \mathrm{~d}$ and at weaning, the mortality percentage was similar $(\mathrm{P}>0.05)$ between males and females lambs; however, lambs born from single lambing had lower $(\mathrm{P}<0.01)$ mortality percentage compared with those from multiple lambing. At lambing, sire breed did not affect $(\mathrm{P}>0.05)$ size, weight or survival rate of litter per lambed ewe, but at weaning, averages for these productivity traits were higher $(\mathrm{P}<0.01)$ in ewes mated to Ka rams than in those mated to Pb rams. Also at weaning, ewes mated to Dr rams had lower $(\mathrm{P}<0.05)$ litter size and survival rate, and similar $(\mathrm{P}>0.05)$ litter weight than ewes mated to Ka rams. Ewes from multiple lambing presented higher $(\mathrm{P}<0.01)$ litter size and weight, and lower $(\mathrm{P}<0.01)$ litter survival rate than those from single lambing during the pre-weaning period. In conclusion, the $\mathrm{Pb}$ ewe productivity synchronized with progesterone and PMSG, and growth of its offspring, can be improved using crossbreeding schemes involving rams from the breeds Dorper and Katahdin.
\end{abstract}

Palabras claves: crecimiento predestete, cruzamientos, ovinos de pelo, peso al destete.

Key words: pre-weaning growth, crossbreeding, hair sheep, weaning weight.

\section{INTRODUCCIÓN}

La demanda de carne ovina se ha incrementado en todo el mundo durante las últimas dos décadas ${ }^{1}$. En este sentido, la lana dejó de ser el objetivo de producción en la mayoría de los sistemas ovinos (Morand-Fehr y Boyazoglu 1999). En México, las razas de lana, que antes eran altamente cotizadas, están siendo desplazadas por razas de pelo que garantizan una producción relativamente constante de cordero a través del año, y con ello, la disponibilidad de carne.

Aceptado: 23.06.2011.

* Instituto de Ciencias Agrícolas, UABC, carretera Delta S/N, Ejido Nuevo León, Mexicali, B.C., CP. 21705, México; lar62@uabc.edu.mx

1 FAO. 2005. Estadísticas producción ovina. http://faostat.fao.org/ site/339/default.aspx
Existen diversas razas de pelo en el país, pero los ovinos raza Pelibuey $(\mathrm{Pb})$ son los que predominan al distribuirse por todo el territorio mexicano bajo diferentes tipos de climas. Esta raza se caracteriza por ser rústica, altamente adaptable a diferentes ambientes, de fácil manejo (Avendaño-Reyes y col 2004) y reproductivamente activa la mayor parte del año. Aunque se observa una reducción en la presencia de ovejas en estro entre los meses de febrero a mayo, no se considera anestro estacional como ocurre en razas de lana (Arroyo y col 2007). Sin embargo, algunos estudios mencionan que la productividad (tamaño y peso de camada al destete) de las ovejas $\mathrm{Pb}$ es menor a la observada en razas de aptitud cárnica o de lana (Segura y col 1996, Wildeus 1997). Diferentes factores se han relacionado con esta menor productividad en las ovejas $\mathrm{Pb}$, entre los que destacan: presencia de un período de baja actividad reproductiva, prolificidad considerada como regular (1,1 a 1,4 crías 
por hembra parida), alta mortalidad de corderos en los primeros días de vida como consecuencia de bajos pesos al nacimiento y una lenta tasa de crecimiento predestete (Galina y col 1996, Segura y col 1996). Tomando como base esta problemática, se han realizado varios estudios para implementar estrategias que ayuden a incrementar la productividad de esta raza y a mantenerla durante todo el año en forma constante. Estas estrategias consisten en el uso de protocolos de sincronización del estro a base de progestágenos y PMSG (Rosado y col 1998, GonzálezReyna y col 1999, Avendaño-Reyes y col 2007), y en el establecimiento de programas de mejoramiento genético, tales como los esquemas de cruzamiento con razas especializadas en producción de carne (Hinojosa-Cuéllar y col 2009, Macías-Cruz y col 2009). Así, a través de la aplicación de estas hormonas exógenas se ha logrado inducir el estro en la época natural de baja actividad reproductiva, así como mejorar la tasa de ovulación, y por ende, la cantidad de partos múltiples, la prolificidad y la fecundidad (Avendaño-Reyes y col 2007). Por su parte, los esquemas de cruzamiento de ovejas $\mathrm{Pb}$ con machos de aptitud cárnica han contribuido a que las crías nazcan con pesos más altos y presenten mayor tasa de crecimiento predestete y peso al destete (Wildeus 1997).

Recientemente, machos de raza Dorper (Dr) y Katahdin (Ka) se introdujeron a los rebaños del país para realizar cruzamientos con las ovejas $\mathrm{Pb}$. La información referente a este cruzamiento es escasa. Asimismo, poco se ha documentado sobre la combinación de programas de sincronización con esquemas de cruzamiento como una alternativa para incrementar la productividad de los rebaños de ovejas $\mathrm{Pb}$. Un estudio reportó una prolificidad de 2,0 crías por oveja parida en ovejas $\mathrm{Pb}$ tratadas con un progestágeno sintético y PMSG (Macías-Cruz y col 2009), además de que las ovejas montadas naturalmente por machos Ka o Dr tuvieron mayor peso de camada por oveja parida $(31,5 \pm 1,9$ vs $26,3 \pm 1,9 \mathrm{~kg})$ y menor mortalidad ( 8 vs $15 \%$ ) al destete que las servidas por machos de su misma raza. En otra investigación reportaron hasta $33 \%$ y $40 \%$ mayor peso al nacimiento y ganancia diaria de peso predestete, respectivamente, en corderos híbridos de $\mathrm{Pb}$ con $\mathrm{Dr}$ o Ka que en los $\mathrm{Pb}$ puro (Hinojosa-Cuéllar y col 2009). No obstante, este resultado no fue constante a través de los años estudiados (2001 a 2005).

Por otra parte, poco se ha hecho para caracterizar el desarrollo de las crías del nacimiento al destete en razas ovinas de pelo y sus híbridos, así como para determinar posibles factores que influyen sobre el crecimiento y mortalidad predestete de las crías de estas razas. Es necesario integrar este tipo de información dentro de la industria ovina del país para hacer más eficientes los sistemas de producción de la especie. Por lo tanto, el objetivo de este estudio fue evaluar el tamaño y peso de camada por ovejas $\mathrm{Pb}$ parida después de ser sometidas a un programa de sincronización con progestágenos y esquemas de cruzamiento terminal con machos Dr, Ka y Pb bajo condiciones estabuladas. Un segundo objetivo fue evaluar el crecimiento predestete de las crías producto de estos cruzamientos.

\section{MATERIAL Y MÉTODOS}

\section{LUGAR DE ESTUDIO}

El estudio se realizó con una base de datos generada a partir de registros reproductivos y productivos de ovejas paridas y crías nacidas entre el 2005 y 2008, producto de un programa de esquemas de cruzamiento terminal entre ovejas $\mathrm{Pb}$ y machos $\mathrm{Dr}, \mathrm{Ka}$ y $\mathrm{Pb}$. Esta información se obtuvo de la unidad ovina del Centro de Bachillerato Tecnológico Agropecuario \#41, el cual se ubica en el Valle de Mexicali, Baja California, al noroeste de México. La región se caracteriza por presentar condiciones climáticas áridas con temperaturas máximas y mínimas promedio anuales de $31,7^{\circ} \mathrm{C}$ y $15,9^{\circ} \mathrm{C}$, respectivamente (García 1987). Las temperaturas máximas y mínimas que se registran en verano e invierno son $50^{\circ} \mathrm{C}$ y bajo $0{ }^{\circ} \mathrm{C}$, respectivamente. La precipitación media anual es de $84,5 \mathrm{~mm}$ y se presenta en los meses de noviembre y diciembre.

\section{BASE DE DATOS}

La base de datos concentró información de 499 crías nacidas a partir de 253 ovejas multíparas de raza $\mathrm{Pb}$ que fueron montadas naturalmente por machos de razas Dr, $\mathrm{Ka}$ y $\mathrm{Pb}$. Para cada cría se registró: fecha de nacimiento, tipo de nacimiento (sencillo, doble o $\geq 3$ (3 y 4 crías)), sexo (macho o hembra), genotipo ( $\mathrm{Dr} \times \mathrm{Pb}, \mathrm{Ka} \times \mathrm{Pb}$ y $\mathrm{Pb}$ puro), fecha de muerte (en caso que haya sucedido) y pesos al nacimiento y al destete. Mientras que de las madres se registró: fecha de servicio, fecha de parto y tipo de parto. A partir de esta información se calcularon variables de estudio del período predestete: ganancia diaria de peso promedio (GDP) del nacimiento al destete y mortalidad acumulada a $30 \mathrm{~d}$ postparto y al destete. También se calcularon variables de productividad de la oveja: tamaño de camada (número de corderos vivos) y peso de camada (sumatoria de los pesos individuales que registraron los corderos vivos de cada camada) al nacimiento y al destete, y tasa de sobrevivencia (número de corderos vivos entre número de corderos nacidos en la camada) a $30 \mathrm{~d}$ postparto y al destete. Todas las variables de productividad fueron calculadas por oveja parida.

\section{MANEJO DEL REBAÑO}

Durante el período de estudio (2005 a 2008) el rebaño se mantuvo bajo un sistema de producción intensivo con un parto por año y en estabulación. Los corrales estuvieron totalmente techados, con piso de tierra y provistos de comederos y bebederos. La alimentación, tanto para hembras, machos y corderos, consistía en una dieta a base de heno de pasto sudan y de alfalfa ad libitum. Cabe mencionar 
que las crías no recibían ningún alimento especial (creep feeding) durante su desarrollo predestete. La disponibilidad de agua fue constante y a libre acceso.

En relación al manejo reproductivo, el grupo de hembras se programaba para que pariera una vez por año. Además, las montas se organizaban usando un esquema de cruzamiento terminal previamente diseñado entre las hembras $\mathrm{Pb}$ y machos de razas $\mathrm{Dr}$, Ka y $\mathrm{Pb}$. En promedio se utilizaron tres machos de cada raza por encastes. La época de encaste en todos los años de estudio se estableció entre los meses de julio y agosto, después de la época de baja actividad reproductiva (enero a mayo) que presentan de manera natural las ovejas de raza de pelo (Arroyo y col 2007).

Durante la época de encaste, las ovejas se sometían a un programa de sincronización del estro que consistía en colocar intravaginalmente una esponja impregnada con $30 \mathrm{mg}$ de un progestágeno sintético (FGA, acetato de fluorogestona; Intervet International BV, Boxmeer Holland) por $12 \mathrm{~d}$. Previo al retiro de la esponja (48 h), se administraba intramuscularmente una dosis de 250 UI de PMSG (hormona gonadotrópica del suero de la yegua preñada; Folligon, Intervet International BV, Boxmeer Holland). Una vez terminado el protocolo de sincronización, las ovejas se dividían aleatoriamente en tres grupos, uno por cada raza de macho a utilizar. Se usó un sistema de encaste controlado para garantizar que todas las hembras que presentaran estro fueran montadas por su respectivo macho y evitar el desgaste de éstos. Dicho sistema consistía en exponer a cada hembra a dos montas, una al momento de mostrar el estro y otra $12 \mathrm{~h}$ después. Finalmente, en la época de partos las ovejas paridas y sus crías se separaban del resto del grupo y se colocaban en otro corral adyacente. Dentro de las primeras $24 \mathrm{~h}$ postparto, las crías se identificaban con muescas, asimismo, se les registraba la información referente a fecha de nacimiento, peso al nacimiento, sexo, tipo de parto, identificación de la madre y genotipo. Posteriormente, los corderos se pesaban individualmente en forma mensual hasta el destete (90 d). También se registraron los corderos muertos.

\section{ANÁLISIS ESTADÍSTICO}

Todas las variables de estudio se sometieron a análisis de normalidad con el procedimiento PROC UNIVARIATE. Para determinar la normalidad de los datos se consideraron los estadísticos descriptivos, histogramas y la prueba Shapiro-Wilk con un $\mathrm{P}>0,05$. El tamaño de camada al nacimiento y al destete y la tasa de sobrevivencia a los $30 \mathrm{~d}$ postparto y al destete presentaron una distribución no normal $(\mathrm{P}<0,05)$, por lo cual estos datos fueron transformados usando raíz cuadrada de $\mathrm{x}+0,5$ para normalizar los datos.

La información fue sometida a análisis de varianza con el procedimiento PROC GLM utilizando un diseño de bloques completamente al azar con arreglo factorial. En las variables de crecimiento predestete de los corderos se utilizó un modelo que incluyó los efectos fijos año de parto (como factor de bloqueo), genotipo del progenitor $(\mathrm{Pb}, \mathrm{Ka}$ y $\mathrm{Dr}$ ), tipo de nacimiento (sencillo, doble y $\geq 3$ crías), sexo (macho y hembra) y las posibles interacciones entre los factores. Para las variables de productividad de la oveja se utilizó un modelo que incluyó el efecto de año como factor de bloqueo y los efectos fijos raza del macho, tipo de parto y la interacción raza $\times$ tipo de parto. Las medias ajustadas fueron comparadas por medio de pruebas "t" Student a una P $\leq 0,05$ usando la opción PDIFF. Cabe mencionar que las medias presentadas en los resultados de los datos transformados corresponden a las generadas con los datos originales. El porcentaje de mortalidad a los $30 \mathrm{~d}$ y al destete se analizó con la prueba de chi-cuadrado a través del procedimiento PROC CATMOD. Todos los procedimientos estadísticos utilizados se desarrollaron con el paquete estadístico SAS versión 9.12 para Windows (SAS Institute, Cary, NC, USA).

\section{RESULTADOS}

A través de los años de estudio se observó que 100\% de las ovejas respondieron al protocolo de sincronización, presentando signos de estro dentro de las primeras $48 \mathrm{~h}$ posteriores al retiro de la esponja y, consecuentemente, el $100 \%$ de las ovejas fueron servidas por monta natural. Por su parte, la fertilidad varió de 90 a 100\%, independientemente de la raza de macho usada para proporcionar el servicio.

La GDP y el peso al destete de los corderos fueron afectados por raza del progenitor $(\mathrm{P}<0,01$; cuadro 1$)$ y la interacción tipo de nacimiento $\times$ sexo $(\mathrm{P}<0,05)$. El peso al nacimiento de los corderos fue similar $(\mathrm{P}>0,05)$ entre los diferentes genotipos, pero los corderos de cruza $\mathrm{Dr}$ presentaron mayor $(\mathrm{P}<0,05)$ peso al destete y GDP que los corderos $\mathrm{Pb}$ puros o de cruza Ka. Entre corderos $\mathrm{Pb}$ puros y cruzas de Ka no se observaron diferencias estadísticamente significativas $(\mathrm{P}>0,05)$ para peso al destete y GDP. La interacción tipo de nacimiento $\times$ sexo afectó significativamente el peso al nacimiento (figura 1), peso al destete (figura 2) y la GDP (figura 3). Al nacimiento, los machos nacidos en parto sencillo presentaron los más altos pesos $(\mathrm{P}<0,05)$, y tanto machos como hembras nacidas en parto $\geq 3$ presentaron los pesos más bajos $(\mathrm{P}<0,05)$. Dentro de los corderos nacidos en parto doble o $\geq 3$, el peso al nacimiento fue similar entre machos y hembras $(P>0,05)$, sin embargo, dentro de los corderos nacidos en parto sencillo los machos fueron más pesados que las hembras $(\mathrm{P}<0,05)$. El peso al destete y la GDP fueron mayores en machos nacidos en parto sencillo $(\mathrm{P}<0,05)$, y más baja en machos y hembras nacidos en parto $\geq 3(\mathrm{P}<0,05)$. Dentro de los corderos nacidos en parto sencillo, los machos fueron más pesados al destete y ganaron más peso por día que las hembras $(\mathrm{P}<0,05)$; misma situación se observó cuando se compararon machos y hembras nacidos en parto doble $(\mathrm{P}=0,05)$. Tanto machos como hembras nacidos en parto $\geq 3$ tuvieron similar peso al destete y GDP $(\mathrm{P}>0,05)$. 
Cuadro 1. Efecto de genotipo del progenitor sobre peso corporal $(\mathrm{kg})$, ganancia diaria de peso $(\mathrm{g})$ y mortalidad $(\%)$ de los corderos durante el período predestete.

Effect of ram genotype on body weight $(\mathrm{kg})$, daily weight gain $(\mathrm{g})$ and mortality $(\%)$ of lambs during the pre-weaning period.

\begin{tabular}{|c|c|c|c|c|c|c|}
\hline \multirow{2}{*}{$\begin{array}{l}\text { Raza del } \\
\text { progenitor }\end{array}$} & \multirow{2}{*}{$\mathrm{n}^{1}$} & \multicolumn{2}{|c|}{ Peso corporal (media $\pm \mathrm{EE})$} & \multirow{2}{*}{$\begin{array}{c}\text { Ganancia } \\
\text { diaria de peso } \\
(\text { media } \pm \mathrm{EE})\end{array}$} & \multicolumn{2}{|c|}{ Mortalidad* } \\
\hline & & Nacimiento & Destete $^{2}$ & & $30 \mathrm{~d}$ & Destete $^{2}$ \\
\hline Dorper & 219 & $2,9 \pm 0,05^{\mathrm{a}}$ & $17,8 \pm 0,33^{\mathrm{a}}$ & $164 \pm 3,43^{\mathrm{a}}$ & $11,4(25 / 219)^{\mathrm{a}}$ & $20,1(44 / 219)^{\mathrm{b}}$ \\
\hline Katahdin & 92 & $2,9 \pm 0,09^{\mathrm{a}}$ & $16,7 \pm 0,50^{\mathrm{b}}$ & $152 \pm 5,20^{\mathrm{b}}$ & $6,5(6 / 92)^{\mathrm{a}}$ & $7,6(7 / 92)^{\mathrm{a}}$ \\
\hline Pelibuey & 188 & $2,8 \pm 0,05^{\mathrm{a}}$ & $15,8 \pm 0,35^{\mathrm{b}}$ & $143 \pm 3,65^{b}$ & $13,3(25 / 188)^{\mathrm{a}}$ & $22,9(43 / 188)^{b}$ \\
\hline
\end{tabular}

Letras diferentes dentro de columna $\left({ }^{\mathrm{a}, \mathrm{b}}\right)$ indican diferencias significativas a $\mathrm{P}<0,05$.

$1 \quad \mathrm{n}=$ Número total de corderos por cada genotipo.

2 El destete se realizó a los 90 d postparto.

* $\quad$ Mortalidad = animales muertos/total de animales

$\mathrm{EE}=$ Error estándar.

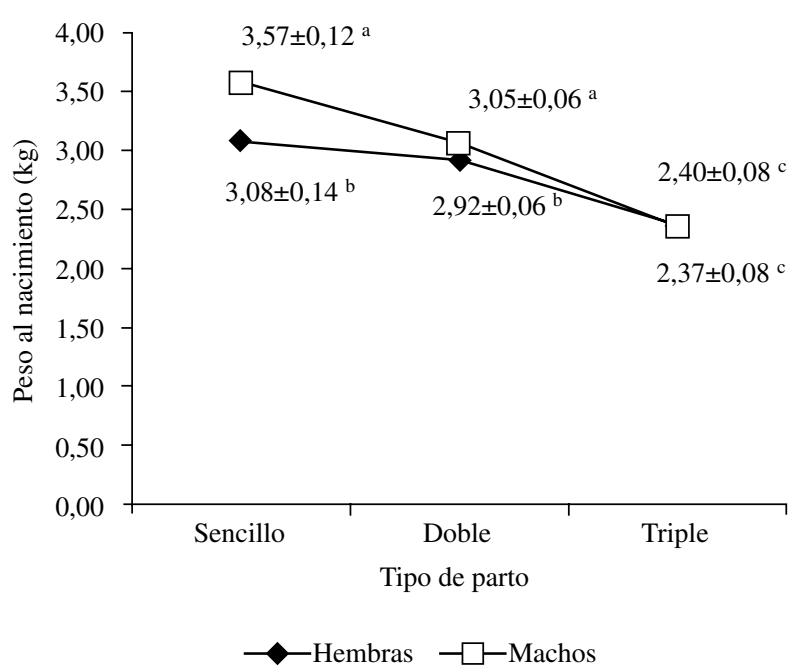

Figura 1. Peso al nacimiento en corderos machos y hembras procedentes de diferente tipo de parto (letras diferentes indican diferencias significativas a $\mathrm{P}<0,05)$.

Birth weight in male and female hair lambs produced from different lambing type (letters different indicate significant difference to $\mathrm{P}<0.05$ ).

El porcentaje de corderos muertos a los $30 \mathrm{~d}$ postparto no fue afectado por raza del progenitor $(\mathrm{P}>0,05)$ (cuadro 1), no obstante se observó que los corderos $\mathrm{Pb}$ puros y $\mathrm{Dr} \times$ $\mathrm{Pb}$ tendieron a morir en mayor medida comparado con los corderos $\mathrm{Ka} \times \mathrm{Pb}$. Al destete $(\mathrm{P}=0,0914)$, los corderos $\mathrm{Ka} \times \mathrm{Pb}$ presentaron más bajo porcentaje de mortalidad que los corderos $\mathrm{Pb}$ puros $(\mathrm{P}<0,01)$ y $\mathrm{Dr} \times \mathrm{Pb}$, entre los cuales no se observaron diferencias significativas en la proporción de corderos muertos $(\mathrm{P}>0,05)$. En relación al efecto de tipo de nacimiento y de sexo sobre el porcentaje de mortalidad de corderos (cuadro 2), se observó que en corderos nacidos en parto sencillo, el porcentaje de mortalidad a los $30 \mathrm{~d}$ postparto fue menor comparado con corderos nacidos en parto doble $\mathrm{o} \geq 3(\mathrm{P}<0,05)$. A $\operatorname{los} 30 \mathrm{~d}$ postparto fue similar el porcentaje de mortalidad

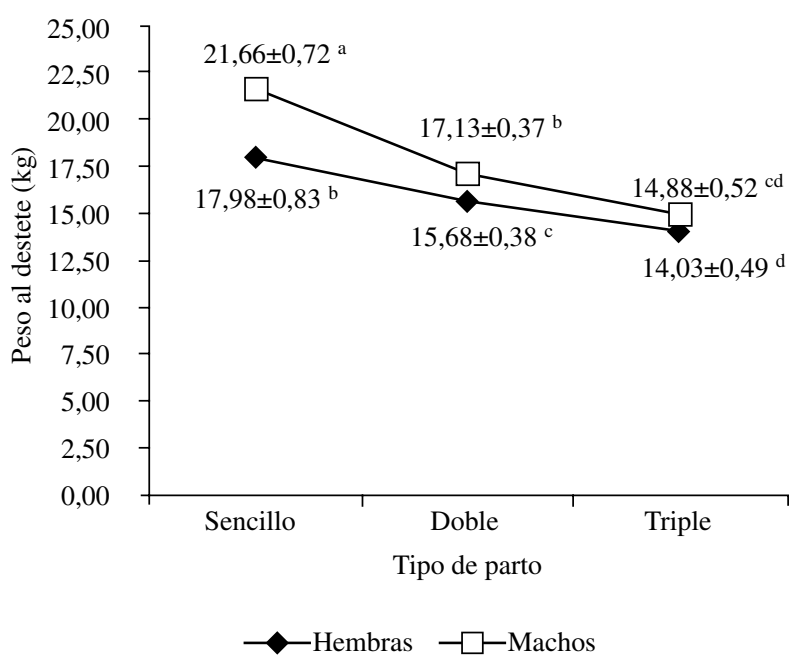

Figura 2. Peso al destete en corderos machos y hembras procedentes de diferente tipo de parto (letras diferentes indican diferencias significativas a $\mathrm{P}<0,05)$.

Weaning weight in male and female hair lambs produced from different lambing type (letters different indicate significant difference to $P<0.05$ ).

entre corderos nacidos en parto doble $y \geq 3$ ( $\mathrm{P}>0,05)$. $\mathrm{Al}$ destete, los corderos nacidos en parto sencillo y doble presentaron similar porcentaje de mortalidad $(\mathrm{P}>0,05)$, pero este tipo de corderos en relación a los nacidos en parto $\geq 3$ tuvieron menor porcentaje de mortalidad $(\mathrm{P}<0,05)$. El porcentaje de mortalidad entre machos y hembras fue similar $(\mathrm{P}>0,05)$.

Los resultados de tamaño de camada, tasa de sobrevivencia y peso de camada por efecto de raza del progenitor y de tipo de parto se presentan en el cuadro 3 . El efecto de la raza paterna no fue significativo para tamaño y peso de camada al nacimiento $(\mathrm{P}>0,05)$, y para tasa de sobrevivencia a los $30 \mathrm{~d}$ postparto. No obstante, al destete, el tamaño y la tasa de sobrevivencia de las camadas fueron mayores $(\mathrm{P}<0,05)$ en ovejas apareadas 


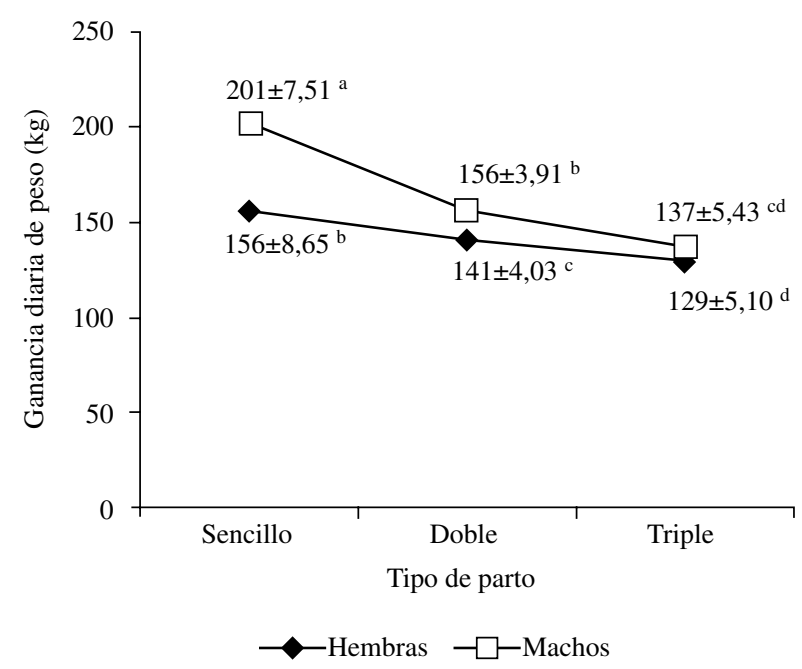

Figura 3. Ganancia diaria de peso en corderos machos y hembras procedentes de diferente tipo de parto (letras diferentes indican diferencias significativas a $\mathrm{P}<0,05)$.

Daily weight gain in male and female hair lambs produced from different lambing type (letters different indicate significant difference to $\mathrm{P}<0.05$ ). con machos Ka que en ovejas apareadas con machos $\mathrm{Dr}$ o $\mathrm{Pb}$, entre las cuales no se observaron diferencias significativas $(\mathrm{P}>0,05)$ para estas variables de estudio. Adicionalmente, el peso de camada al destete fue similar $(\mathrm{P}>0,05)$ entre ovejas apareadas por machos Dr y Ka, pero estas ovejas tuvieron camadas más pesadas $(\mathrm{P}<0,01)$ que las ovejas apareadas con machos $\mathrm{Pb}$. Con respecto al efecto de tipo de parto, se observó que el tamaño y peso de camada al nacimiento y al destete fue más alto $(\mathrm{P}<0,01)$ en ovejas de tipo de parto $\geq 3$ que en ovejas de parto doble y sencillo, asimismo, las ovejas de parto doble presentaron valores promedios más altos $(\mathrm{P}<0,01)$ para estas variables de estudio que las ovejas de parto sencillo. La tasa de sobrevivencia a los $30 \mathrm{~d}$ postparto fue mayor $(\mathrm{P}<0,05)$ en camadas de ovejas de parto sencillo que en camadas de ovejas de tipo de parto $\geq 3$. Entre camadas de parto sencillo y doble no se observaron diferencias significativas $(\mathrm{P}>0,05)$ en tasa de sobrevivencia a los $30 \mathrm{~d}$, al igual que entre camadas de parto doble $\mathrm{y} \geq 3$. Al destete, el tipo de parto de la oveja no afectó $(\mathrm{P}>0,05)$ la tasa de sobrevivencia de la camada.

Cuadro 2. Efecto de tipo de nacimiento y sexo sobre el porcentaje de mortalidad* de corderos durante el período predestete. Effect of birth type and sex on mortality of lambs during the pre-weaning period.

\begin{tabular}{lcccccc}
\hline \multirow{2}{*}{$\begin{array}{l}\text { Período } \\
\text { Predestete }\end{array}$} & \multicolumn{3}{c}{ Tipo de parto } & & \multicolumn{2}{c}{ Sexo } \\
\cline { 2 - 5 } \cline { 3 - 5 } & Sencillo & Doble & $\geq 3$ & Macho & Hembra \\
\hline $30 \mathrm{~d}$ & $3,4(2 / 59)^{\mathrm{a}}$ & $10,4(30 / 287)^{\mathrm{b}}$ & $15,7(24 / 153)^{\mathrm{b}}$ & & $12,5(32 / 256)^{\mathrm{a}}$ & $9,9(24 / 243)^{\mathrm{a}}$ \\
Destete $^{1}$ & $15,2(9 / 59)^{\mathrm{a}}$ & $17,0(49 / 287)^{\mathrm{a}}$ & $23,5(36 / 153)^{\mathrm{b}}$ & & $17,2(44 / 256)^{\mathrm{a}}$ & $20,6(50 / 243)^{\mathrm{a}}$ \\
\hline
\end{tabular}

Letras diferentes dentro de hileras $\left(^{\mathrm{a}, \mathrm{b}}\right)$ indican diferencias significativas a $\mathrm{P}<0,05$.

1 El destete se realizó a los 90 d post-parto.

* $\quad$ Mortalidad = animales muertos/total de animales.

Cuadro 3. Efecto de raza del progenitor y del tipo de parto sobre tamaño de camada, sobrevivencia y peso de camada de las ovejas Pelibuey durante el período predestete.

Effect of ram breed and lambing type on litter size, survival and litter weight of Pelibuey ewes during the pre-weaning period.

\begin{tabular}{|c|c|c|c|c|c|c|c|}
\hline & \multirow{2}{*}{$\mathrm{n}^{1}$} & \multicolumn{2}{|c|}{ Tamaño de camada ${ }^{2}($ media \pm EE $)$} & \multicolumn{2}{|c|}{ Sobrevivencia $^{3}($ media $\pm \mathrm{EE})$} & \multicolumn{2}{|c|}{ Peso de camada ${ }^{4}($ media $\pm E E)$} \\
\hline & & Nacimiento & Destete $^{5}$ & $30 \mathrm{~d}$ & Destete $^{5}$ & Nacimiento & Destete $^{5}$ \\
\hline \multicolumn{8}{|c|}{ Raza del progenitor } \\
\hline Dorper & 111 & $2,0 \pm 0,06^{\mathrm{a}}$ & $1,6 \pm 0,07^{\mathrm{a}}$ & $0,90 \pm 0,03^{\mathrm{a}}$ & $0,82 \pm 0,03^{\mathrm{a}}$ & $5,6 \pm 0,16^{\mathrm{a}}$ & $27,9 \pm 1,33^{\mathrm{a}}$ \\
\hline Katahdin & 44 & $2,1 \pm 0,11^{\mathrm{a}}$ & $1,9 \pm 0,11^{\mathrm{b}}$ & $0,95 \pm 0,04^{\mathrm{a}}$ & $0,94 \pm 0,06^{\mathrm{b}}$ & $5,5 \pm 0,25^{\mathrm{a}}$ & $30,1 \pm 2,14^{\mathrm{a}}$ \\
\hline Pelibuey & 98 & $1,9 \pm 0,07^{\mathrm{a}}$ & $1,5 \pm 0,07^{\mathrm{a}}$ & $0,89 \pm 0,03^{\mathrm{a}}$ & $0,80 \pm 0,04^{\mathrm{a}}$ & $5,4 \pm 0,17^{\mathrm{a}}$ & $23,2 \pm 1,41^{b}$ \\
\hline \multicolumn{8}{|c|}{ Tipo de parto } \\
\hline Sencillo & 59 & $1,0 \pm 0,02^{\mathrm{a}}$ & $0,9 \pm 0,09^{\mathrm{a}}$ & $0,98 \pm 0,03^{\mathrm{a}}$ & $0,90 \pm 0,05^{\mathrm{a}}$ & $3,4 \pm 0,21^{\mathrm{a}}$ & $18,5 \pm 1,80^{\mathrm{a}}$ \\
\hline Doble & 144 & $2,0 \pm 0,02^{b}$ & $1,7 \pm 0,06^{\mathrm{b}}$ & $0,91 \pm 0,02^{\mathrm{ab}}$ & $0,87 \pm 0,03^{\mathrm{a}}$ & $5,9 \pm 0,15^{\mathrm{b}}$ & $28,6 \pm 1,24^{\mathrm{b}}$ \\
\hline$\geq 3$ & 50 & $3,1 \pm 0,02^{\mathrm{c}}$ & $2,4 \pm 0,09^{c}$ & $0,85 \pm 0,03^{\mathrm{b}}$ & $0,81 \pm 0,05^{\mathrm{a}}$ & $7,1 \pm 0,20^{\mathrm{c}}$ & $34,0 \pm 1,76^{\mathrm{c}}$ \\
\hline
\end{tabular}

Letras diferentes dentro de columna $\left({ }^{a, b, c}\right)$ indican diferencias significativas a $\mathrm{P}<0,05$.

$\mathrm{n}=$ Número de ovejas Pelibuey paridas.

Promedio del número de corderos vivos por camada.

Promedio del número de corderos vivos por camada / número de corderos nacidos por camada.

4 Expresado en $\mathrm{kg}$.

5 El destete se realizó a los 90 d postparto.

$\mathrm{EE}=$ Error estándar. 


\section{DISCUSIÓN}

El protocolo de sincronización basado en el uso de esponjas intravaginales y 250 UI de PMSG mostró ser eficiente para sincronizar el estro de las ovejas $\mathrm{Pb}$ en un período de tiempo relativamente corto ( $48 \mathrm{~h}$ postretiro de la esponja) a través de los años de estudio. Además, juzgado por la fertilidad (90-100\%), se demuestra que las esponjas vaginales en combinación con monta natural en ovejas Pelibuey pueden mejorar la eficiencia reproductiva de los rebaños del noroeste de México. Aun cuando existen investigaciones (Contreras-Solis y col 2009, Gatti y col 2010) en las cuales se menciona que la monta natural después de tratar las ovejas con progestágenos intravaginales conlleva a una reducción en la fertilidad de los rebaños ovinos debido a alteraciones en el transporte de los espermatozoides y cambios fisicoquímicos en el aparato reproductor de la oveja. El porcentaje de ovejas en estro y la fertilidad observada en este estudio son consistentes con resultados previamente publicados en ovejas $\mathrm{Pb}$ en la zona de estudio (Avendaño-Reyes y col 2007, MacíasCruz y col 2009).

Uno de los motivos de realizar esquemas de cruzamiento entre hembras $\mathrm{Pb}$ y machos de aptitud cárnica (Ka y Dr) es incrementar el peso al nacimiento y, consecuentemente, disminuir el porcentaje de mortalidad en los primeros días postparto en corderos de raza $\mathrm{Pb}$. Sin embargo, los resultados de este estudio mostraron que el peso al nacimiento fue estadísticamente similar entre corderos $\mathrm{Pb}, \mathrm{Dr} \times \mathrm{Pb}$ y $\mathrm{Ka} \times \mathrm{Pb}$. Posiblemente, los corderos de cruza Dr o Ka no presentaron mayor peso al nacimiento que los $\mathrm{Pb}$ debido a que el desarrollo de éstos durante la etapa fetal fue limitado por el ambiente y espacio uterino considerando que las ovejas $\mathrm{Pb}$ son de talla pequeña, comparadas con las de raza de lana. El genotipo del cordero interacciona con el ambiente uterino para que se exprese el potencial genético de crecimiento en los fetos (Michels y col 2000). No obstante, durante el período predestete se observó que los corderos $\mathrm{Dr} \times \mathrm{Pb}$ presentaron mayor crecimiento comparado con los otros genotipos, siendo más marcado en relación a los $\mathrm{Pb}$ puros, lo cual se debió a que la raza $\mathrm{Pb}$ se caracteriza por presentar lenta tasa de crecimiento, ya que genéticamente no ha sido sometida a una rigurosa selección para rasgos de crecimiento (Macías-Cruz y col 2010). Adicionalmente, un posible efecto de heterosis en conjunto con el potencial que posee la raza Dr para crecimiento, pueden explicar el mejor peso al destete y GDP observados en corderos de cruza Dr. No obstante, en este estudio no se puede delimitar realmente cuánto influyó la heterosis porque no se tiene información de las razas Dr y $\mathrm{Ka}$ en forma pura. En concordancia con estos resultados han reportado que el peso al destete y el crecimiento predestete de los corderos $\mathrm{Pb}$ pueden ser mejorados a través de esquemas de cruzamiento entre ovejas $\mathrm{Pb}$ y machos de razas de lana (Bores y col 2002) o pelo (Hinojosa-Cuéllar y col 2009, Macías-Cruz y col 2009) especializadas en producción de carne. Por otra parte, la ausencia de diferencias en mortalidad de corderos a los $30 \mathrm{~d}$ postparto entre los genotipos estudiados es un reflejo de los resultados de peso al nacimiento por efecto de genotipo. Esto debido a que el peso al nacimiento se correlaciona negativamente con la tasa de mortalidad de corderos (Gardner y col 2007). Sin embargo, en general durante todo el período predestete se observó que los corderos $\mathrm{Ka} \times \mathrm{Pb}$ presentaron menor tasa de mortalidad que los corderos $\mathrm{Pb}$ puros y de cruza con Dr. Similarmente, Macías-Cruz y col (2009) reportaron que los corderos $\mathrm{Pb}$ puros y $\mathrm{Dr} \times \mathrm{Pb}$ se morían en mayor proporción comparados con $\mathrm{Ka} \times \mathrm{Pb}$, y ellos lo relacionaron con la vigorosidad de las crías al nacimiento, habilidad para consumir alimentos sólidos a una edad más temprana y la capacidad de adaptación a las condiciones climáticas. En este contexto, las variaciones en la tasa de mortalidad y crecimiento predestete entre genotipos bajo un ambiente determinado denotan la necesidad de mantener una diversidad de razas considerando que cada raza puede tener un ambiente diferente para sobrevivir y mantener una producción óptima (Steinheim y col 2008).

En este estudio, la interacción tipo de nacimiento $x$ sexo influyó en el peso al nacimiento de los corderos, el cual fue mayor en machos nacidos en parto sencillo que en machos y hembras nacidos en parto doble $y \geq 3$, al igual que en hembras nacidas en parto sencillo. Adicionalmente, tanto machos y hembras nacidos en partos $\geq 3$ tuvieron los pesos más bajos. Lo anterior se observa frecuentemente y puede deberse a las diferencias que existen en desarrollo gestacional entre productos de diferente sexo, el cual es favorable para machos (Hinojosa-Cuéllar y col 2009); así como también a la competencia por los nutrientes y espacio uterino cuando se desarrolla más de un producto dentro de la oveja (Gardner y col 2007). En ovinos, la tasa de crecimiento del esqueleto durante la gestación es más rápida en machos que en hembras debido a la hormona testosterona que es producida abundantemente por fetos machos (de Zegher y col 1998). Acorde con estos resultados, otros estudios (González y col 2002, Avendaño y col 2004, Macías-Cruz y col 2009) realizados con razas de pelo también reportaron menor peso al nacimiento en corderos producto de partos múltiples y en hembras comparado con los machos. Por otra parte, la GDP y peso al destete también fueron afectados por la interacción tipo de nacimiento $\times$ sexo, observándose similares resultados a los encontrados para peso al nacimiento. Por lo tanto, el peso al nacimiento es un factor que explica parcialmente que los corderos machos nacidos en parto sencillo, y los corderos machos y hembras nacidos en parto $\geq 3$ hayan mostrado más altas y más bajas, respectivamente, GDP y peso al destete. Esta relación entre peso al nacimiento y crecimiento predestete ha sido observada en otros estudios de ovinos, tanto en razas de lana (Benyi y col 2006) como de pelo (Carrillo y Segura 1993, Macedo y Arredondo 2008, Hinojosa-Cuéllar y col 2009). Otros factores a considerar para explicar estos resultados son: 
1) el consumo de calostro y leche por cordero nacido es menor a medida que se incrementa el tamaño de camada (Gardner y col 2007), conllevando a que crías nacidas en partos múltiples presenten menor tasa de crecimiento y peso al destete que crías nacidas en parto único; y 2) los machos producen hormona testosterona, la cual ayuda a incrementar el desarrollo muscular, y por ende, favorece el aumento del peso vivo al destete (Hafez 2004). Ambos factores, consumo de leche y producción de testosterona, ocasionaron que corderos machos nacidos en parto sencillo tuvieran superior tasa de crecimiento. Varios estudios hechos en razas de pelo coinciden en mencionar que a medida que se incrementa el número de corderos nacidos por oveja, la tasa de crecimiento y peso al destete se reducen (González y col 2002, Avendaño y col 2004, Aksakal y col 2009, Hinojosa-Cuéllar y col 2009). Estos mismos estudios también reportan una diferencia en crecimiento predestete y peso al destete a favor de los machos. Sin embargo, en otro estudio realizado bajo el mismo manejo y clima que el presente estudio, no encontraron diferencia debido a sexo sobre el crecimiento predestete de corderos $\mathrm{Pb}$ puros y cruzados con Dr y Ka (Macías-Cruz y col 2009). Una posible variación entre años de estudio puede ser la causa de estas diferencias.

Los resultados de mortalidad por efecto de tipo de parto y de sexo son consistentes con los publicados en la literatura para este tipo de cruzamiento (Macías-Cruz y col 2009). Asimismo, la mayor mortalidad observada a medida que se incrementa el tamaño de camada coincide también con resultados encontrados para ovinos de otras razas, tanto de pelo (Galina y col 1996), de lana (Christley y col 2003) o sus cruzas (Cloete y col 2007). En un estudio donde identificaron los posibles factores de riesgo relacionados con la mortalidad de corderos, reportaron que los principales factores causantes son el peso al nacimiento y el consumo de leche por cordero (Christley y col 2003). De tal manera que a medida que el peso al nacimiento es más bajo y el número de crías nacidas por oveja parida se incrementa, la probabilidad de que mueran los corderos es mayor, lo cual explica la mayor tasa de mortalidad observada en corderos de parto múltiple. Tanto machos como hembras presentaron similar tasa de mortalidad a los $30 \mathrm{~d}$ postparto y al destete. Resultados similares fueron reportados por Cloete y col (2007).

La producción de cordero por oveja parida al destete es un reflejo de la capacidad reproductiva y de la habilidad materna de la oveja, así como del potencial genético que tienen las crías para crecer rápidamente. Las características de productividad de las ovejas (tamaño, peso y sobrevivencia de la camada) son de mayor importancia biológica y económica para el producto que observar de forma separada el comportamiento reproductivo del rebaño y el crecimiento de las crías (Snowder y Fogarty 2009). En este estudio se observó que el tamaño de camada por oveja $\mathrm{Pb}$ parida no varió por efecto de raza paterna, lo cual se debe a que esta variable depende de la capacidad ovulatoria de la raza de la oveja y a la habilidad de dichas ovejas para llevar a buen término la gestación (Menéndez y col 2004). Cabe señalar que el número de crías por oveja al parto fue mayor (2,0 vs 1,3 crías) al reportado (Avendaño-Reyes y col 2004) comúnmente para ovejas $\mathrm{Pb}$ bajo climas desérticos. Esto se debe a que en este estudio las ovejas fueron sincronizadas y tratadas con PMSG, hormona que estimula el desarrollo folicular y, por ende, la tasa ovulatoria. Los resultados de productividad de la oveja también muestran que las camadas de ovejas apareadas con machos $\mathrm{Dr}$, $\mathrm{Ka}$ y $\mathrm{Pb}$ tuvieron en promedio $5,5 \mathrm{~kg}$ de peso al nacimiento y $92 \%$ de tasa de sobrevivencia a los $30 \mathrm{~d}$ postparto, sin efecto de raza paterna. El resultado de peso de camada al nacimiento se atribuye a que tampoco se encontró efecto de raza paterna sobre peso individual de los corderos y el tamaño de camada al nacimiento. Por su parte, el resultado de la tasa de sobrevivencia es un reflejo del peso al nacimiento y del porcentaje de mortalidad a los $30 \mathrm{~d}$ postparto encontrado por efecto de genotipo. Al destete, la tasa de sobrevivencia fue mayor en camadas hijas del macho Ka como consecuencia de la menor tasa de mortalidad observada en corderos de cruza Ka $(7,6 \%)$, comparado con la de corderos $\mathrm{Pb}$ puros $(20,1 \%)$ y cruzas de $\operatorname{Dr}(22,9 \%)$. Lo anterior se reflejó en camadas más numerosas y pesadas al destete en ovejas apareadas con machos Ka. Menéndez y col (2004) destacan la importancia de producir camadas numerosas para incrementar el peso de la camada y, por ende, la productividad de la oveja. Cabe mencionar que las camadas de ovejas apareadas con machos Dr presentaron similar peso al destete que las de ovejas apareadas con Ka, no obstante que el tamaño y tasa de sobrevivencia de la camada fue superior en estas últimas ovejas. Posiblemente esto se deba al mayor peso al destete observado en cordero de cruza Dr por efecto de la heterosis. Varios estudios han reportado un incremento en el peso de la camada al destete por efecto de realizar esquemas de cruzamiento, atribuyéndolo al efecto de heterosis (Segura y col 1996, Cloete y col 2007, Snowder y Fogarty 2009). En adición a estos resultados, un estudio previo (Macías-Cruz y col 2009) donde cruzaron las mismas razas usadas en esta investigación, encontraron que las camadas de machos Dr y Ka fueron alrededor de $17 \%$ más pesadas al destete que las de macho $\mathrm{Pb}$. Cabe mencionar que la información disponible sobre productividad de las ovejas $\mathrm{Pb}$ bajo este esquema de cruzamiento es escasa, por lo que la comparación de los resultados con la obtenida en otros trabajos es limitada. Sin embargo, estos resultados muestran la importancia de realizar esquemas de cruzamiento de ovejas $\mathrm{Pb}$ con machos de aptitud cárnica para mejorar la sobrevivencia de los corderos y, por tanto, la productividad de la oveja en cuanto al número y peso de corderos destetados se refiere.

La productividad de las ovejas $\mathrm{Pb}$ bajo condiciones estabuladas está altamente relacionada con el tipo de parto. Las camadas producto de parto único presentaron mayor tasa de sobrevivencia a los $30 \mathrm{~d}$, pero menor tamaño y 
peso de camada durante todo el período predestete en comparación con las camadas nacidas en parto múltiple. Estos resultados sugieren que para incrementar la productividad de una oveja $\mathrm{Pb}$ es necesario tener tamaños de camadas de más de una cría. No obstante, varios estudios coinciden en mencionar que aun y cuando se presenten partos dobles, triples o hasta cuádruples, no es una garantía de una mayor productividad por oveja si no se tiene extremo cuidado con las crías, ya que las posibilidades de muerte de estos corderos incrementa con el tamaño de camada al nacimiento (Menéndez y col 2004, Snowder y Fogarty 2009). Dicha mortalidad se registra principalmente en los primeros días postparto (Menéndez y col 2004), lo cual explica que las camadas de parto múltiple presentaron $13 \%$ menos tasa de sobrevivencia a los $30 \mathrm{~d}$ postparto que las camadas de parto sencillo. Sin embargo, es preciso remarcar que el tamaño de camada de los corderos de nacimiento múltiple fue muy superior ( 2,0 corderos por oveja parida en promedio) al observado en camadas de parto sencillo o doble a lo largo del período predestete. Este resultado sugiere que las ovejas estuvieron produciendo suficiente leche para mantener dos crías. Además, que el efecto de heterosis y el manejo pre y postparto en estabulación ofrecido a las ovejas y sus crías fueron condiciones favorables que ayudaron a las ovejas de parto múltiple a mantener la sobrevivencia de la mayoría de sus crías. Acorde con los resultados de efecto de tipo de parto, en ovejas Blackbelly y Pelibuey han observado mayor productividad en ovejas de parto doble que en sencillo (Segura y col 1996).

Se concluye que las ovejas Pelibuey en condiciones de manejo intensivo pueden incrementar su productividad (tasa de sobrevivencia y peso de la camada) al destete a través de esquemas de cruzamiento con machos de razas especializadas en producción de carne como Dorper y Katahdin. Asimismo, bajo este tipo de cruzamiento, los corderos Pelibuey pueden mejorar su crecimiento predestete. Adicionalmente, la productividad de las ovejas Pelibuey y el crecimiento de sus crías están influenciados principalmente por el tipo de parto. Es conveniente que bajo un plan nutricional adecuado las ovejas sean sincronizadas con FGA y PMSG para incrementar el tamaño de camada, lo cual conlleva a la producción de camadas más pesadas.

\section{RESUMEN}

El objetivo de este estudio fue evaluar la productividad de 253 ovejas Pelibuey $(\mathrm{Pb})$ paridas entre el 2005 y 2008, las cuales fueron apareadas con machos Pb, Dorper (Dr) y Katahdin (Ka) después de haberles sincronizado el estro con progesterona y PMSG. Adicionalmente se evaluaron el peso al nacimiento y al destete y la ganancia diaria de peso predestete (GDP) de 499 crías producto de este cruzamiento. Un modelo lineal que incluyó efectos de bloque (año de parto), raza del padre, tipo de parto, sexo y sus interacciones fue usado para analizar el crecimiento predestete. El mismo modelo, sin incluir efecto de sexo, fue aplicado para analizar la productividad de la oveja. El peso al nacimiento y el porcentaje de mortalidad a los $30 \mathrm{~d}$ postparto fueron similares $(\mathrm{P}>0,05)$ entre los genotipos evaluados en corderos, pero el peso al destete y la GDP fueron mayores $(\mathrm{P}<0,05)$ en corderos de cruza Dr que en corderos $\mathrm{Pb}$ puros o de cruza Ka. La interacción tipo de parto $\times$ sexo afectó $(\mathrm{P}<0,05)$ el peso al nacimiento y el crecimiento predestete de los corderos. A los $30 \mathrm{~d}$ y al destete, el porcentaje de mortalidad fue similar $(\mathrm{P}>0,05)$ entre corderos machos y hembras, sin embargo, los corderos nacidos en parto único tuvieron menor $(\mathrm{P}<0,01)$ porcentaje de mortalidad comparado con los de parto $\geq 3$. Al parto, raza del macho no afectó $(\mathrm{P}>0,05)$ el tamaño, peso y tasa de sobrevivencia de la camada por oveja parida, pero al destete, las medias de estos rasgos de productividad fueron mayores $(\mathrm{P}<0,01)$ en ovejas apareadas por machos Ka que en ovejas apareadas por machos $\mathrm{Pb}$. También al destete, las ovejas apareadas por machos $\mathrm{Dr}$ tuvieron menor $(\mathrm{P}<0,05)$ tamaño y tasa de sobrevivencia de camada, pero similar peso de camada $(\mathrm{P}>0,05)$ que las ovejas apareadas por machos Ka. Las ovejas de parto múltiple presentaron mayor tamaño y peso de camada, y menor tasa de supervivencia que las ovejas de parto único durante el período predestete. En conclusión, la productividad de las ovejas $\mathrm{Pb}$ sincronizadas con progestágenos y PMSG y el crecimiento de sus crías pueden ser mejorados usando esquemas de cruzamiento que involucren machos Dorper y Katahdin.

\section{REFERENCIAS}

Aksakal V, M Macit, N Esenbuga. 2009. Effects of various ages of weaning on growth characteristics, survival rate and some body measurements of Awassi lambs. J Anim Vet Adv 8, 1624-1630.

Arroyo J, J Gallegos-Sánchez, A Villa-Godoy, JM Berruecos, G Perera, J Valencia. 2007. Reproductive activity of Pelibuey and Suffolk ewes at $19^{\circ}$ north latitude. Anim Reprod Sci 102, 24-30.

Avendaño-Reyes L, FD Álvarez-Valenzuela, S Quintero, L Molina, FJ Cisneros. 2004. Assessment of some productive traits of the Pelibuey sheep in northwestern Mexico: Preliminary results. Cuban J Agr Sci 38, 129-134.

Avendaño-Reyes L, FD Álvarez-Valenzuela, L Molina-Ramírez, R Rangel-Santos, A Correa-Calderón, J Rodríguez-García, M CruzVillegas, PH Robinson, TR Famula. 2007. Reproductive performance of Pelibuey ewes in response to estrus synchronization and artificial insemination in Northwestern Mexico. J Anim Vet Adv 6, 807-812.

Benyi K, D Norris, N Karbo, KA Kgomo. 2006. Effects of genetic and environmental factors on pre-weaning and post-weaning growth in West African crossbred sheep. Trop Anim Health Prod 38, 547-554.

Bores QRF, PA Velázquez, M Heredia. 2002. Evaluación de razas terminales en esquemas de cruza comercial con ovejas de pelo F1. Téc Pec Méx 40, 71-79.

Carrillo L, JC Segura. 1993. Environment and genetic effects on preweaning growth performance of hair sheep in México. Trop Anim Health Prod 25, 173-178.

Christley RM, KL Morgan, THD Parkin, NP French. 2003. Factors related to the risk of neonatal mortality, birth weight and serum immunoglobulin concentration in lambs in the UK. Prev Vet Med $57,209-226$.

Cloete JJE, SWP Cloete, JJ Olivier, LC Hoffman. 2007. Terminal crossbreeding of Dorper ewes to Ile de France Merino Landsheep and SA Mutton Merino sires: ewe production and lamb performance. Small Rumin Res 69, 28-35.

Contreras-Solis I, B Vasquez, T Diaz, C Letelier, A Lopez-Sebastian, A Gonzalez-Bulbes. 2009. Efficiency of estrous synchronization in tropical sheep by combining short-interval cloprostenol-based protocols and "male effect". Theriogenology 71, 1018-1025.

de Zegher F, I Francois, ALM Boehmer, G Saggese, JMO Hiort, C Sultan, P Clayton, R Brauner, E Cacciari, L Ibéñez, G Van Vliet, A Tiulpakov, N Saka, M Ritzén, WG Sipell. 1998. Androgens and fetal growth. Horm Res 50, 243-244.

Galina MA, R Morales, E Silva, B López. 1996. Reproductive performance of Pelibuey and Blackbelly sheep under tropical management systems in México. Small Rumin Res 22, 31-37. 
García E. 1987. Modificaciones al sistema de clasificación climática de Koeppen. Editorial Universidad Nacional Autónoma de México, México, Pp 12-13.

Gardner DS, PJ Buttery, Z Daniel, ME Symonds. 2007. Factors affecting birth weight in sheep: maternal environment. J Reprod Fertil 133, 297-307.

Gatti M, P Zunino, R Ungerfeld. 2010. Changes in the aerobic vaginal bacterial mucous load after treatment with intravaginal sponges in anoestrous ewes: effect of medroxiprogesterone acetate and antibiotic treatment use. Reprod Dom Anim, doi: 10.1111/j.1439-0531.2010.01626.x

González GR, G Torres, M Castillo. 2002. Growth of Blackbelly lambs between birth and final weight in the humid tropics of México. Vet Méx 33, 443-453.

González-Reyna A, E Márquez-García, H Lizárraga-Tracy, JC MartínezGonzález. 1999. Dose response effects of PMSG on ovulation rate and folicular development in Pelibuey ewes treated with Syncromate-B implants. Small Rumin Res 31, 149-155.

Hafez ESE. 2004. Reproducción e inseminación artificial en animales. Editorial McGraw-Hill, México, Pp 33-55.

Hinojosa-Cuéllar JA, FM Regalado, J Oliva. 2009. Crecimiento prenatal y predestete en corderos Pelibuey, Dorper, Katahdin y sus cruces en el sureste de México. Rev Cient FCV LUZ 19, 522-532.

Macedo R, V Arredondo. 2008. Efecto del sexo, tipo de nacimiento y lactancia sobre el crecimiento de ovinos Pelibuey en manejo intensivo. Arch Zootec 57, 219-228.

Macías-Cruz U, FD Álvarez-Valenzuela, A Correa-Calderón, L MolinaRamírez, A González-Reyna, S Soto-Navarro, L Avendaño-Reyes. 2009. Pelibuey ewe productivity and subsequent pre-weaning lamb performance using hair-sheep breed under a confinement system. $J$ Appl Anim Res 36, 255-260.
Macías-Cruz U, FD Álvarez-Valenzuela, J Rodríguez-García, A Correa-Calderón, NG, Torrentera-Olivera, L Molina-Ramírez, L Avendaño-Reyes. 2010. Crecimiento y características de canal en corderos Pelibuey puros y cruzados F1 con razas Dorper y Katahdin en confinamiento. Arch Med Vet 42, 147-154.

Menéndez BA, G Alexandre, N Mandonnet. 2004. Discussion on the importance, definition and genetic components of the number of animals born in the litter with particular emphasis on small ruminants in tropical conditions. Small Rumin Res 54, 1-11.

Michels H, E Decuypere, O Onagbesan. 2000. Litter size, ovulation rate and prenatal survival in relation to ewe body weight: genetics review. Small Rumin Res 38, 199-209.

Morand-Fehr P, J Boyazoglu. 1999. Present state and future outlook of the small ruminant sector. Small Rumin Res 34, 175-188.

Rosado J, E Silva, MA Galina. 1998. Reproductive management of hair sheep with progesterone and gonadotropins in the tropics. Small Rumin Res 27, 237-242.

SAS INSTITUTE. 2004. SAS/STAT: user's guide statistics released 9. $12^{\text {th }}$ ed. SAS Institute Inc., Cary, N.C., USA.

Segura JC, L Sarmiento, O Rojas. 1996. Productivity of Pelibuey and Blackbelly ewes in Mexico under extensive management. Small Rumin Res 21, 57-62.

Snowder GD, NM Fogarty. 2009. Composite trait selection to improve reproduction and ewe productivity: a review. Anim Prod Sci 49, 9-16.

Steinheim G, J Ødegård, T Ådnøy, G Klemetsdal. 2008. Genotype by environment interaction for lamb weaning weight in two Norwegian sheep breeds. J Anim Sci 86, 33-39.

Wildeus S. 1997. Hair sheep genetic resources and their contribution to diversified small ruminant production in the United States. J Anim Sci 75, 630-640. 